IJBPAS, December, 2019, 8(12): 2261-2269

ISSN: 2277-4998

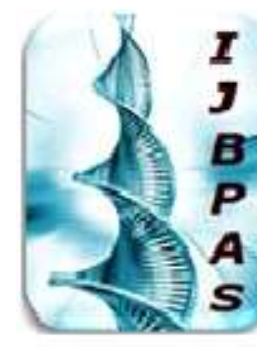

International Journal of Biology, Pharmacy

and Allied Seiences (IJBPAS)

'A Bridge Betusen Caboratory and Q ando'

WwW.ijbpas.com

GRAPHENE SYNTHESIS: A REVIEW

KHAN MU ${ }^{1,2^{*}}$, KHAN $K^{3}$, RAZA $S^{4}$, SADIA $H^{5}$, USMAN $O^{6}$ AND ULLAH $W^{7}$

${ }^{1}$ Department of Chemical engineering, University of Engineering and Technology, Peshawar

${ }^{2}$ School of Chemical engineering and Technology, Tianjin University China

${ }^{3}$ Department of Environmental Science, International Islamic University, Islamabad, Pakistan

${ }^{4}$ Department of Food Science \& Technology, UCP (University of Central Punjab, Lahore)

${ }^{5}$ Department of Biotechnology, University of Information Technology, Engineering and

Management Sciences Quetta

${ }^{6}$ Department of Physics, University of Lahore, Pakistan

${ }^{7}$ Environmental Science COMSATS University Islamabad Abbottabad Campus Pakistan

"Corresponding Author: Maaz Ullah Khan

Received $20^{\text {th }}$ April 2019; Revised 20 ${ }^{\text {th }}$ May 2019; Accepted 22 ${ }^{\text {nd }}$ June 2019; Available online $1^{\text {st }}$ Dec. 2019

https://doi.org/10.31032/IJBPAS/2019/8.12.4850

ABSTRACT

Graphene has achieved a great amount of popularity and interest from the science world because of its extraordinary physical, mechanical and thermal properties. Graphene is an allotrope of carbon, having one-atom-thick planar sheets of sp2 bonded carbon atoms densely pack Edina honeycomb crystal lattice many methods to synthesize graphene have been developed over a short period and we believe it is necessary to create a list of the most notable approaches. This article focuses on the methods to synthesize graphene in an attempt to summarize and document advancements in the synthesis of graphene research and future prospects.

Keywords: Graphene, graphene; synthesis; nanomaterials; graphite; 2D; biomass INTRODUCTION

Graphene is perhaps the simplest material ever produced. It has one-atom-thick form of carbon and definitely the thinnest planar sheets of $\mathrm{sp} 2$ bonded carbon atoms 
densely packed in a honeycomb crystal lattice (Setchedin et al., 2007). It is useful to understand graphene as a few layers of graphite. In this context, the extraordinary properties of honeycomb carbon are not surprising. In middle ages, the layered morphology and weak dispersion forces between adjacent sheets were utilized to make marking instruments, similar to our use of pencils today. Recently, graphite is being used as dry lubricant, instead of lavishly priced hexagonal boron nitride and molybdenum disulphide (Allen et al., 2009). Graphite is also used in electrodes because of high in-plane electrical conductivity $(\sim 104 / \Omega \cdot \mathrm{cm})$ and in industrial blast furnaces because of its excellent thermal conductivity $(\sim 3000 \mathrm{~W} / \mathrm{m} \cdot \mathrm{K})$ (Enoki, et al., 2008). The term 'graphene' was first used by Boehm et al. while describing single layer carbon foils. It was derived from graphite and the suffix 'end'. Graphite flakes are basically a stack of graphene layers with an interplanar spacing of $3.5^{\circ} \mathrm{A}$ and having- $\mathrm{C}$ bond length of about $0.142{ }^{\circ} \mathrm{A}$ explained by (Geim et al., 2010). "Graphene is a single atomic plane of graphite, which and this is essential - is sufficiently isolated from its environment to be considered free-standing". Graphene strikes as a remarkable candidate for making functional nanocomposites having excellent properties. The fact that its precursor (graphite) is readily available makes it more tempting. Defect free graphene presents outstanding physical properties, such as high intrinsic mobility and ballistic transport, high thermal conductivity and Young's modulus, an optical transmittance of almost $98 \%$ and a large specific surface area (Castillo-Martínez et al., 2011). Much attention has been given to the characterization of graphene and its derivatives, so we will not be discussing the properties of graphene. This review article focuses on the brief history and recent advancement in synthesis of graphene.

\section{Synthesis of graphene}

Graphene has been in the spotlight for quite a while and numerous analysts have been chipping away at graphene combination, therefore, a few strategies have been accounted for the shedding of graphite into graphene. They can be partitioned into two principle classes: the top-down methodology and the base up methodology. Graphite is just a stack suspended on a micrometer-estimate metallic framework. (tubes consideration (d) Raman spectra of graphite, GO and graphene sheets (Krishnamoorthy et al, 2012). (republished with consent). of graphene layers and to have the option to isolate these layers into individual sheets of graphene, van der Waals 
powers should be survived. This methodology is alluded to as the top-down methodology. A few difficulties are related with this methodology, similar to surface imperfections that happen during sheet detachment and the isolated sheets deagglomerating a short time later. All in all, the top-down methodologies offer low yields and are repetitive techniques (Warner, J. $\mathrm{H}$ et al 2013). While the top-down methodology centers around breaking graphene forerunner (graphite) into nuclear layers from a stack base up methodology actualizes carbon particles as structure squares; commonly, these carbon atoms are gotten from elective sources. Even however the base up methodology isn't reasonable to create graphene sheets with huge surface region, this methodology offers the likelihood of assembling graphene Nano-strips and graphene specks (supposed nanoflakes) in enormous amounts.

\section{Top down approach}

\section{Mechanical approach}

Mechanical or micromechanical exfoliation method has been a turning point in the history of graphene. It is still the key synthesis technique for obtaining high quality graphene for research purposes since it delivers high quality graphene films sized from 5 to $10 \mu \mathrm{m}$ as it has been witnessed by
TEM and AFM analysis. However, the uneven thickness of lobtailed by this method and high production cost due to low yield render this method unusable for mass production. These methods are a prime example of the top-down approach, where a graphene precursor (graphite, GO, etc.) is taken apart layer through layer, forming graphene sheets. The group from Manchester University including the two Nobel Prize winners, Geim and Novoselov, obtained graphene by micro-mechanical alleviation of graphite. They used 'the scotch tape' or peel off method to repeatedly split graphite crystals into increasingly thinner pieces (Warner, J. H.et al 2014).

\section{Graphite intercalation}

Intercalation. Distinctive synthetic species can be embedded between graphite interlayers petroproduct graphite intercalation mixes (GIC). Because of the nearness of these intercalants between graphite layers, the separation between them increments. This likewise changes the properties of graphene in light of the fact that the expanded interlayer separation influences electronic coupling between graphene layers. (Leeet al. 2014) have examined the impacts of intercalation on capacitance in a cathode and have revealed improved electro- 
conductivity of $\mathrm{MnO}_{2}$ because of the nearness of GIC.

\section{Nanotube scling}

Carbon nanotubes have as antecedent for graphene. Graphene nanoribbons which are a couple of microns in size can be acquired by this methodology. Hypothetically, carbon nanotubes could be sliced open to frame 2D graphene sheets (Soldano, et al., 2015). A few techniques have been created, similar to plasma scratching of nanotubes mostly installed in polymer film or putting carbon nanotubes in an answer containing potassium permanganate and sulfuric corrosive. Top notch graphene can be acquired from nanotube cutting. (Chen et al., 2016) built up a nanotube cutting strategy which is appropriate for mechanical use. Carbon nanotubes were rubbed between ground-glass surface deracinating heap of $0.06 \mathrm{~N} / \mathrm{cm}^{2}$ making erosion cut them into graphene sheets. Graphene acquired by this methodology can be utilized in applications, for example, FET, interconnects, NEM and composites. In any case, given then stove forth forerunner (CNT), this strategy probably won't be financially suitable for business creation.

\section{Pyrolysis method}

Pyrolysis offers simplicity of procedure and can be scaled up for generation. Be that as it may, the yield is low and there are polluting influences in the final item. In this strategy, a 1:1 molar proportion of sodium $(2 \mathrm{~g})$ and ethanol $(5 \mathrm{~mL})$ are warmed in a fixed reactor vessel at $220^{\circ} \mathrm{C}$ for 72 hours to get the graphene forerunner: strong solvothermal item. It is then quickly pyrolyzed and the rest of the item washed with deionized water $(100 \mathrm{~mL})$. The suspended strong is then vacuum filtered and dried in a vacuum stove at $100^{\circ} \mathrm{C}$ for 24 hours. This solvothermal response technique can convey graphene yield of approx.0.1gper1mLofethanol-ordinarily yielding $0.5 \mathrm{~g}$ per response (Warner, et al., 2015). Super capacitors, sensors, and so forth. Decrease of GO has by and large been favored for graphene decrease due to the lower level of peeling accomplished by graphite and expandable graphite. GO can be shed by sonication in water however the subsequent material is electrically protecting, so it ought to be decreased to reestablish its conductivity. Decrease can be performed by synthetic or warm treatment. Fast warming has additionally been accounted for as a conceivable arrangement bringing about exceptional returns of scattered carbon powder with a couple of percent of carbon 
flakes Likewise, by scattering oxidized and synthetically handled graphite in water, and utilizing paper-production strategies, the monolayer flakes in a type of a solitary sheet reinforced in all respects capably can be acquired. These sheets, called graphene oxide paper, have a deliberate malleable modulus of 32 GA Graphene based layers are impermeable to all gases and fluids apart from water, which dissipates through the film, as it didn't exist.

\section{Electrochemical exfoliation}

(Parvez et al., 2016) revealed a fascinating strategy for shedding graphene from graphite utilizing an electrochemical technique. They utilized $0.1 \quad \mathrm{M} \quad \mathrm{H}_{2} \mathrm{SO}_{4}$ arrangement as electrolyte, while graphite flakes as anode and platinum wires as cathode. A positive charge of $+10 \mathrm{~V}$ was utilized to the framework and graphite flakes started to disintegrate in the arrangement. Following 2 minutes, the voltage predisposition was evacuated, and the shed graphitic material was gathered by vacuum filtration. The example was then washed with water over and over to guarantee expulsion of remaining acidic substance. The acquired powder was scattered in DMF, bringing about EG sheets (Warner, et al, 2016) have proposed a one-pot technique to blend diverse nanocarbon frames, including graphene, by peeling of graphite in ionic fluids. They explored different avenues regarding joining of 1-butyl-3methylimidazolium tetra flouroborate [BMIm] [BF4] in water utilized as an electrolyte for the electrochemical peeling of graphite. Static possibilities of 1.5 to $15 \mathrm{~V}$ were connected. The peeled particles were washed with water and ethanol to accomplish a nonpartisan $\mathrm{pH}$.

\section{Sonication}

Brilliant, un-oxidized graphite and graphene flakes can be gotten by sonication. This strategy is a prime case of the top-down methodology towards graphene amalgamation since, in this technique, ultrasonic vitality is utilized to isolate graphene layers stacked together in a forerunner. Be that as it may, this technique requires a lot of vitality since sonication is the main vitality source and it tends to be a worry, while scaling up this strategy (Warner, et al., 20218). Additionally, evacuation of pollutions is an issue that happens in this procedure. Graphene gotten by this system can be utilized in the field of polymer fillers, straightforward anodes and sensors. Graphene can be scattered in specific solvents, like N-methyl-pyrrolidone, at centralizations of up to $0.01 \mathrm{mg} / \mathrm{mL}$. Such arrangements can be utilized in an assortment 
of procedures to fuse graphene in polymers and composites, for example, splash covering, vacuum filtration, dissolvable throwing or drop throwing (Brumfiel et al., 2017) While sonication introduces a simple methodology towards making graphene, it postures difficulties for industrialization as far as power utilization. Dissolvable helped sonication is a basic modification of sonication strategy and a conspicuous advance ahead. Graphene is gotten from graphite through sonication with the assistance of a dissolvable (NMP, TEA, and so on.), where graphene can be isolated from graphite with the assistance of centrifugation. NMP or other ionic fluid can be utilized in such procedure [50, 51]. A solitary cycle yield of up to $5 \%$ is realistic from this procedure and around $70 \%$ of the graphene acquired by this procedure is $1 \mathrm{~nm}$ thick having electrical conductivity of up to 5000 $\mathrm{S} \cdot \mathrm{m}-1$ [52]. When utilizing the dissolvable supported frameworks, graphene keeps an eye on re-stack together after sonication as a result of van der Waals powers. To maintain a strategic distance from such issues, surfactants or scattering specialists can be included the arrangement before sonication, so the graphene sheets would note-stack. This strategy can be utilized to get graphene sheets from graphite without concoction modification. It additionally offers graphene yields as high as $5.33 \mathrm{mg} / \mathrm{mL}$. On account of the utilization of an ionic fluid (1-hexyl-3methyl-imidazolium hexafluorophosphate or $\mathrm{HMIH}$ ), this procedure is steady and green [51]. Sodium dodecylbenzene sulfonate (SDBS) likewise will in general balance out graphene layers and keeps them from accumulation [53]. Liu et al. have revealed a technique utilizing GO suspension (by means of sonication) to get $3 \mathrm{D}$ hydrogel as a help for microorganism immobilization and BOD biosensor. Nonpartisan Red (NR) arrangement was included into $20 \mathrm{~mL}$ of 2 $\mathrm{mg} / \mathrm{mL}$ GO suspension, which was sonicated further for $10 \mathrm{~min}$ and then the solution was kept at $180^{\circ} \mathrm{C}$ for $5 \mathrm{~h}$ in a teflon-lined autoclave, trailed by temperature decrease to room temperature. The got hydrogel was washed completely with deionized water before portrayal.

\section{Ball milling}

On a fundamental level, ball processing is a sub-class of mechanical peeling, yet numerous varieties of this technique have been grown, so this strategy has the right to be talked about independently. Ball processing has been being used for blending and molecule measure decrease for quite a while. Researchers have utilized this molecule 
estimate decrease way to deal with shed graphene sheets from graphite, in some cases utilizing attractive help (Dreyer et al., 2017). And here and there utilizing compound or dissolvable help. Attractive help gives filtration of metallic particles present in the antecedents, while synthetic help keeps the sheets from re-stacking utilizing substance connections, for example, $\pi-\pi$ Such strategies give FLGs layers) and offer high generation yield.

\section{Dry ice method}

As per dry ice technique, graphene can be acquired by consuming $3 \mathrm{~g}$ of $\mathrm{Mg}$ lace inside a dry ice bowl, concealed by another dry ice $\left(\right.$ solidCO $\left.{ }_{2}\right)$ chunk. $\mathrm{Mg}$ should be totally scorched in $\mathrm{CO}_{2}$ and the buildup is mixed medium-term in $100 \mathrm{~mL}$ of $1 \mathrm{M} \mathrm{HCl}$. Both $\mathrm{Mg}$ and $\mathrm{MgO}$ are solvent in water. Along these lines, the blend is filtered and the buildup is washed with deionized water till $\mathrm{pH}$ turns nonpartisan. To evacuate water content, the buildup should be dried mediumterm under vacuum at $100{ }^{\circ} \mathrm{C}$ bringing about a graphene yield of $680 \mathrm{mg}(92 \%)$.

\section{REFERENCES}

[1] Schedin, F., Geim, A. K., Morozov, S. V., Hill, E. W., Blake, P., Katsnelson, M. I., \& Novoselov, K. S. (2007). Detection of individual gas molecules adsorbed on graphene. Nature materials, 6(9), 652.

[2] Allen, M. J., Tung, V. C., \& Kaner, R. B. (2009). Honeycomb carbon: a review of graphene.Chemical reviews, 110 (1), 132-145.

[3] Enoki, T., Suzuki, M., \& Endo, M. (2003). Graphite intercalation compounds and applications. Oxford University Press.

[4] Castillo-Martínez, E., CarreteroGonzález, J., Sovich, J., \& Lima, M. D. (2014). High temperature structural transformations of few layer graphene nanoribbons obtained by unzipping carbon nanotubes. Journal of Materials Chemistry A, 2(1), 221-228.

[5] Geim, A. K. (2009). Graphene: status and prospects. Science, 324 (5934), 1530-1534.

[6] Rao, C. N. R., Biswas, K., Subrahmanyam, K. S., \& Govindaraj, A. (2009). Graphene, the new nanocarbon. Journal of Materials Chemistry, 19(17), 2457-2469.

[7] Soldano, C., Mahmood, A., \& Dujardin, E. (2010). Production, properties and potential of graphene. Carbon, 48(8), 2127-2150. 
[8] Krishnamoorthy, K., Kim, G. S., \& Kim, S. J. (2013). Graphene nanosheets: Ultrasound assisted synthesis and characterization. Ultrasonics sonochemis -try, 20(2), 644-649.

[9] Warner, J. H., Schaffel, F., Rummeli, M., \& Bachmatiuk, A. (2012). Graphene: fundamentals and emergent applications. Newnes.

[10] Lee, H., Kang, J., Cho, M. S., Choi, J. B., \& Lee, Y. (2011). $\mathrm{MO}_{2} /$ graphene composite electrodes for supercapacitors: the effect of grapheme intercalation on capacitance. Journal of Materials Chemistry, 21(45), 18215-18219.

[11] Brumfiel, G. (2009). Nanotubes cut to ribbons new techniques open up carbon tubes to create ribbons. Nature, 15.

[12] Jiao, L., Zhang, L., Wang, X., Diankov, G., \& Dai, H. (2009). Narrow graphene nanoribbons from carbon nanotubes. Nature, $458(7240$ ), 877 .

[13] Choucair, M., Thordarson, P., \& Stride, J. A. (2009). Gram-scale production of graphene based on solvothermal synthesis and sonication. Nature nanotechnology, 4(1), 30.

[14] Weng, W. (2003). G.; He, B.; Wu, C., L. Exfoliation of graphite flake and its nanocomposites. Carbon, 41, 619.

[15] Dreyer, D. R., Park, S., Bielawski, C. W., \& Ruoff, R. S. (2010). The chemistry of grapheme oxide. Chem -ical society reviews, 39, 228-240.

[16] Eswaraiah, V., Aravind, S. S. J., \& Ramaprabhu, S. (2011). Top down method for synthesis of highly conducting graphene by exfoliation of graphite oxide using focused solar radiation. Journal of Materials Chemistry, 21(19), 6800-6803.

[17] Joshi, R. K., Carbone, P., Wang, F. C., Kravets, V. G., Su, Y., Grigorieva, I. V., \& Nair, R. R. (2014). Precise and ultrafast molecular sieving through grapheme oxide membranes. Science, 3 43(6172), 752-754.

[18] Gurunathan, S., Han, J. W., Eppakayala, V., \& Kim, J. H. (2013). Green synthesis of graphene and its cytotoxic effects in human breast cancer cells. International journal of nanomedicine, 8, 1015.

[19] Parvez, K., Li, R., Puniredd, S. R., Hernandez, Y., Hinkel, F., Wang, S., \& Müllen, K. (2013). Electrochemically exfoliated graphene as solution-processable, highly conductive electrodes for 
organic electronics. ACS nano, 7(4), 3598-3606.

[20] Hernandez, Y., Nicolosi, V., Lotya, M., Blighe, F. M., Sun, Z., De, S., \& Boland, J. J. (2008). High-yield production of graphene by liquidphase exfoliation of graphite. Nature nanotechnology, 3(9), 563.

[21] Nuvoli, D., Valentini, L., Alzari, V., Scognamillo, S., Bon, S. B., Piccinini, M., \& Mariani, A.(2011). High concentration few-layer graphene sheets obtained by liquid phase exfoliation of graphite in ionic liquid. Journal of Materials Chemistry, 21(10), 3428-3431.

[22] Liu, L., Zhai, J., Zhu, C., Gao, Y., Wang, Y., Han, Y., \& Dong, S. (2015). One-pot synthesis of 3 dimensional reduced graphene oxide-based hydrogel as support for microbe immobilization and BOD biosensor preparation. Biosensors and Bioelectronics, 63, 483489.

[23] Leon, V., Quintana, M., Herrero, M. A., Fierro, J. L., de la Hoz, A., Prato, M., \& Vazquez, E. (2011). Few-layer graphene's from ballmilling of graphite with melamine. Chemical Communicatio$n s, 47(39)$, 10936-10938.
[24] Lin, T., Tang, Y., Wang, Y., Bi, H., Liu, Z., Huang, F., \& Jiang, M. (2013). Scotch-tape-like exfoliation of graphite assisted with elemental sulfur and graphene-sulfur composites for high-performance lithium-sulfur batteries. Energy \& Environmental Science, 6(4), 12831290. 